\title{
Anther culture in rice proportionally rescues microspores according to gametophytic gene effect and enhances genetic study of hybrid sterility
}

\author{
Yoshitaka Kanaoka ${ }^{1}$, Daichi Kuniyoshi ${ }^{1}$, Eri Inada ${ }^{1}$, Yohei Koide ${ }^{1}$, Yoshihiro Okamoto ${ }^{2}$, Hideshi Yasui ${ }^{3}$ \\ and Yuji Kishima ${ }^{1 *}$ (i)
}

\begin{abstract}
Background: To investigate plant hybrid sterility, we studied interspecific hybrids of two cultivated rice species, Asian rice (Oryza sativa) and African rice (O. glaberrima). Male gametes of these hybrids display complete sterility owing to a dozen of hybrid sterility loci, termed HS loci, but this complicated genetic system remains poorly understood.

Results: Microspores from these interspecific hybrids form sterile pollen but are viable at the immature stage. Application of the anther culture (AC) method caused these immature microspores to induce callus. The segregation distortion of 11 among 13 known HS loci was assessed in the callus population. Using many individual calli, fine mapping of the HS loci was attempted based on heterozygotes produced from chromosome segment substitution lines (CSSLS). Transmission ratio distortion (TRD) from microspores was detected at 6 of $11 \mathrm{HS}$ loci in the callus population. The fine mapping of $S_{1}$ and $S_{19}$ loci using CSSLs revealed precise distances of markers from the positions of HS loci exhibiting excessive TRD.

Conclusions: We demonstrated that AC to generate callus populations derived from immature microspores is a useful methodology for genetic study. The callus population facilitated detection of TRD at multiple HS loci and dramatically shortened the process for mapping hybrid sterility genes.
\end{abstract}

Keywords: Anther culture, Callus, Hybrid sterility, Mapping, Microspore, Oryza sativa, O. glaberrima, Rice

\section{Background}

Methods that maintain immature microspores as living cells can rescue pollen aborted between meiosis and fertilization and enable genetic studies of gametophytic characteristics. Anther culture (AC) is a technique in which plants are regenerated from microspores via callus formation in in vitro culture $[1,2]$. Callus induced from microspores generally retains a haploid genome after meiosis in the parental plant, which can facilitate the characterization of genetic factors regulating the cell

\footnotetext{
${ }^{*}$ Correspondence: kishima@abs.agr.hokudai.ac.jp

${ }^{1}$ Laboratory of Plant Breeding, Research Faculty of Agriculture, Hokkaido University, Sapporo 060-8589, Japan

Full list of author information is available at the end of the article
}

viability of male gametes. Here, we propose a methodology to apply this $\mathrm{AC}$ technique for the study of hybrid sterility (HS).

HS, which is a postzygotic isolation mechanism, typically refers to a deficiency in the reproductive capability of $F_{1}$ hybrids [3]. In rice, HS is a well-known phenomenon that acts as a barrier to interspecific crossing, particularly between Asian rice (Oryza sativa L.) and African rice (O. glaberrima Steud.) $[4,5] . \mathrm{F}_{1}$ hybrids between these two species produce almost no normal pollen, resulting in complete spikelet sterility. This pollen sterility is caused by the abortive effect of $H S$ loci, generally known as $H S$ loci, which reduce pollen and/or embryosac fertility only in heterozygotes [6-16]. Thirteen $H S$ loci causing pollen sterility have been mapped thus far: 
gamete eliminator loci $S_{1}, S_{33}(t)$, and $S_{37}(t)$, which cause abortion of both pollen grains and embryo sacs, and pollen killer loci $S_{3}, S_{18}, S_{19}, S_{20}, S_{21}, S_{29}(t), S_{34}(t), S_{36}(t)$, $S_{38}(t)$, and $S_{39}(t)$, which cause abortion of pollen grains (Table 1) [6-16]. Some of these HS loci induce allele-specific abortion owing to allelic conflicts at the HS locus in a gametophytic manner. For example, sativa-glaberrima $S_{1}$ heterozygotes can only transmit the gamete carrying the $S_{1}^{g}$ allele from O. glaberrima to progenies, while the $S_{1}^{s}$ allele from $O$. sativa cannot participate in fertilization [17]. Such differential transmission between two alleles from their heterozygote is generally referred to as transmission ratio distortion (TRD) and results in segregation distortion (SD) in the progenies $[17,18]$. Both male and female TRD by $S_{1}$ is directly linked to the respective semi-sterility of pollen and embryo sacs in $S_{1}$ heterozygotes. Major pollen sterility in sativa-glaberrima $\mathrm{F}_{1}$ hybrids results from the integration of multiple $H S$ loci that individually may cause only partial sterility [18].

An ultimate goal of research on $H S$ loci is the establishment of a strategy to overcome HS and to facilitate wide crossing for gene introgression or exploitation of heterosis. Two $S_{1}$ locus components have recently been isolated in sativa-glaberrima interspecific hybrids [19, 20]. Dissection of $S_{1}$ at the molecular level has taken over 20 years since its first discovery. A major ratedetermining step in research on pollen killer and gamete eliminator genes in rice interspecific hybrids is successive backcrossing to dilute severe HS; this process, which requires the isolation of an $H S$ locus from a number of
$H S$ loci, is a time-consuming facet of the genetic analysis $[21,22]$. Rapid, efficient methods to identify functional $H S$ loci and isolate additional loci in any cross combination are thus needed.

Here, we propose a new method for rapidly detecting $H S$ loci using $\mathrm{AC}$ of interspecific hybrids. During callus induction from microspores, individual $H S$ loci may exhibit differences in TRD leading to SD in the callus population, similar to observations in regenerated plants [23-25]. This phenomenon might be applicable to the mapping and identification of $H S$ loci using AC calli derived from heterozygotes. Because successive backcrosses would not be required, such a method would drastically shorten the time required for detection and genetic analysis of $H S$ loci.

In this study, we verified that the AC method successfully triggered callus formation in microspores of sativaglaberrima interspecific $\mathrm{F}_{1}$ hybrids and then examined the segregation patterns of known $H S$ loci. Our results allowed us to infer whether the lethal effect of each $H S$ locus due to SD appeared at early microspore developmental stages in the callus population. Finally, we finemapped $S_{1}$ and $S_{19}$ loci using the anther-derived calli of their heterozygotes, which were produced from chromosome segment substitution lines (CSSLs) [26].

\section{Methods}

\section{Plant materials}

For the first experiment, we produced interspecific $F_{1}$ hybrids by crossing O. sativa L. ssp. japonica with $O$.

Table 1 Genotype segregation of markers linked to $S$ loci in anther culture-induced calli derived from interspecific F1 hybrids between 0 . sativa and 0. glaberrima

\begin{tabular}{|c|c|c|c|c|c|c|c|c|}
\hline \multirow[t]{2}{*}{ S loci } & \multirow[t]{2}{*}{ Function $^{a}$} & \multirow[t]{2}{*}{ Chr. } & \multirow[t]{2}{*}{ Markers } & \multicolumn{2}{|c|}{ No. of calli (frequency) } & \multirow[t]{2}{*}{$x^{2}(1: 1)$} & \multirow{2}{*}{$\begin{array}{l}\text { Allele transmitted } \\
\text { preferentially via pollen }\end{array}$} & \multirow[t]{2}{*}{ References } \\
\hline & & & & $s$ & $g$ & & & \\
\hline$S_{1}$ & $\mathrm{Ge}$ & 6 & $\begin{array}{l}\text { RM19359 } \\
\text { RM204 }\end{array}$ & $5(0.10)$ & $47(0.90)$ & $33.92^{* *}$ & $g$ & {$[6,17,34]$} \\
\hline $\mathrm{S}_{3}$ & $P k$ & 11 & RM536 ${ }^{\mathrm{b}}$ & $18(0.35)$ & $34(0.65)$ & $4.92^{*}$ & g & {$[7]$} \\
\hline$S_{18}$ & $P k$ & 10 & $\mathrm{RM} 25321^{\mathrm{b}}$ & $29(0.56)$ & $23(0.44)$ & 0.69 & - & {$[8]$} \\
\hline$S_{19}$ & $P k$ & 3 & $\mathrm{RM} 60^{\mathrm{b}}$ & $9(0.17)$ & $43(0.83)$ & $22.23^{* *}$ & $g$ & {$[9,35]$} \\
\hline$S_{20}$ & $P k$ & 7 & $\mathrm{RM} 82^{\mathrm{b}}$ & $13(0.25)$ & $38(0.75)$ & $12.25 * *$ & $g$ & [8] \\
\hline$S_{21}$ & $P k$ & 7 & $\mathrm{RM} 429^{\mathrm{b}}$ & $47(0.90)$ & $5(0.10)$ & $33.92^{* *}$ & $s$ & {$[8]$} \\
\hline$S_{29}(t)$ & $P k$ & 2 & $\mathrm{RM} 279^{\mathrm{b}}$ & $46(0.88)$ & $6(0.12)$ & $30.77^{* *}$ & - & [11] \\
\hline$S_{34}(t)$ & $P k$ & 3 & RM7 & $26(0.50)$ & $26(0.50)$ & 0.00 & - & [13] \\
\hline$S_{36}(t)$ & $P k$ & 2 & RM207 & $29(0.56)$ & $23(0.44)$ & 0.69 & - & [14] \\
\hline$S_{37}(t)$ & $\mathrm{Ge}$ & 1 & RM449 & $33(0.63)$ & $19(0.37)$ & 3.77 & $g$ & {$[15,37]$} \\
\hline$S_{38}(t)$ & $P k$ & 4 & RM16260 & $27(0.52)$ & $25(0.48)$ & 0.08 & - & [15] \\
\hline
\end{tabular}

Asterisks aberrant segregation to the theoretical ratio $(\mathrm{s}: \mathrm{g}=1: 1)$ : ${ }^{*} \mathrm{p}<0.05 ;{ }^{* *} \mathrm{p}<0.01 .1$

a Ge gamete eliminator, $P k$ pollen killer

b unique markers selected based on the mapping in previous studies shown in Reference column

c s, O. Sativa allele; g, O. glaberrima allele; -, unknown 
glaberrima Steud.. The donor parents, O. glaberrima accessions, Acc. IRGC 104038 from Senegal (designated as WK21) and Acc. IRGC 103777 from Mali (designated as WK18), were kindly provided by the International Rice Germplasm Center, the International Rice Research Institute, Philippines and had been conserved in Kyushu University. The japonica cultivar Nipponbare was reciprocally crossed with $\mathrm{WK} 21$ and $\mathrm{WK} 18$; the resulting $\mathrm{F}_{1}$ lines were named as N/WK21 and WK21/N in the first case and N/WK18 and WK18/N in the second (collectively referred to as $\mathrm{WK} / \mathrm{N})$.

The materials used in the second experiment were produced from two CSSLs, GIL27 and GIL31, which are introgression lines carrying segments from WK21 in the genetic background of $O$. sativa ssp. japonica 'Taichung 65' (T65) (Additional file 1: Fig. S1) [26]. GIL27 and GIL31 were obtained from a set of O. glaberrima introgression lines maintained by Kyushu University under the auspices of the National Bioresource Project. GIL31 and GIL27 carry chromosomal segments from O. glaberrima corresponding to the $H S$ loci $\mathrm{S}_{1}$ and $S_{19}$, respectively (Additional file 1: Fig. S1). To produce $S_{1}$ and $S_{19}$ heterozygotes, GIL31and GIL27 were backcrossed with T65, resulting in GIL31/T65 and GIL27/T65, respectively (collectively referred to as GIL/T65). All strains used in this study were grown according to conventional cultivation methods in a greenhouse at Hokkaido University. Starting at the seven-leaf stage, a short-day treatment (10 h light/14 h dark) was applied to induce reproductive growth.

\section{Anther culture}

The two types of heterozygotes, WK/N and GIL/T65, and their parental lines were subjected to AC. Spikes enveloped by leaf sheaths were sampled at the booting stage. After excision of leaf blades and surface sterilization with $70 \%$ ethanol, the panicles were incubated at $10{ }^{\circ} \mathrm{C}$ (low temperature treatment) in the dark for 4-10 days. Approximately 70 anthers per dish were plated onto N6 [27] (Additional file 2: Table S1a), RI-13 [28] (Additional file 2: Table S1b), or SK-1 [29] (Additional file 2: Table S1c) callus-induction medium (CIM) prepared in a ø $90 \mathrm{~mm} \times \mathrm{H} 15 \mathrm{~mm}$ plastic dish. The plated anthers were hermetically sealed and then cultured at $25^{\circ} \mathrm{C}$ in the dark for 4 months. Growing callus was transplanted to fresh medium to promote further growth.

\section{Classification of microspore developmental stages}

To collect microspores at different developmental stages for use in the $\mathrm{AC}$ experiment, primary rachis-branches of each panicle were separated at the booting stage from top to bottom into three parts: first to third branches, fourth to sixth branches, and sixth to ninth branches, representing late, middle, and early stages, respectively. The youngest and oldest anthers in each group were sampled and fixed with formaldehyde-acetic acid-ethanol solution after $10{ }^{\circ} \mathrm{C}$ incubation. Microspores in the fixed spikelets were stained with acetocarmine, and their developmental stages were determined by optical microscopy as described previously [30]. Five microspore developmental stages, namely, classes A, B, C, D, and E, were defined as follows: A, early uninucleate $(1 \mathrm{~N})$ and earlier stages; $\mathrm{B}$, early $1 \mathrm{~N}$ to middle/late $1 \mathrm{~N}$ stages; $\mathrm{C}$, the middle/late $1 \mathrm{~N}$ stage; $\mathrm{D}$, middle/late $1 \mathrm{~N}$ to binucleate $(2 \mathrm{~N})$ stages; and E, 2N and later stages (Fig. 1).

\section{Genotyping of AC-generated calli and detection of SD}

Genomic DNA was extracted from AC-generated calli of WK/N and GIL/T65 and from leaves of $\mathrm{F}_{2}$ individuals of GIL/T65 following the method of Martin et al. [31] with one modification: calli were ground in elution buffer without freezing. A total of 104 calli induced from WK/N were used for the genotyping analysis. The genotyping was carried out with $11 \mathrm{HS}$-loci-linked simple sequence repeat (SSR) markers showing polymorphism between $O$. sativa and O. glaberrima according to previous studies (cited in Table 1 and Additional file 2: Table S2). PCR amplifications for genotyping were performed using GoTaq Green Master Mix (Promega), with the resulting products subjected to agarose gel electrophoresis. Other genotyping analyses of calli from GIL/T65 were focused on segregation of the SSR markers linked to $S_{1}$ and $S_{19}$ (Additional file 2: Table S2).

The homozygosity and heterozygosity of each callus was judged according to the SSR marker genotyping results. Calli retaining any heterozygous alleles were excluded from further analyses, as a callus with heterozygous alleles might not have been derived from a microspore with a haploid genome. Calli that were homozygous at all markers were subjected to the following genetic analyses. The SD of each marker was evaluated with a Chi square goodness-of-fit test based on a theoretical ratio of $s(s s): g(g g)=1: 1$ under no TRD. The segregation in two $\mathrm{F}_{2}$ populations derived from GIL/T65 plants was evaluated to confirm the gametophytic lethal effects of $S_{1}$ and $S_{19}$. Genomic DNAs isolated from $\mathrm{F}_{2}$ plants were genotyped with SSR markers adjacent to $S_{1}$ and $S_{19}$ loci (Additional file 2: Table S2). The genotype frequency of each marker was assessed by a Chi square test for goodness of fit to the Mendelian ratio (ss:sg:gg=1:2:1). Another test was performed to distinguish the effect of the pollen killer from that of the gamete eliminator. In the case of the complete abortion of only male gametes possessing an $s$ allele at a pollen killer locus, such as $S_{19}$, the expected segregation of this locus would be $s g: g g=1: 1$. The segregation of the 


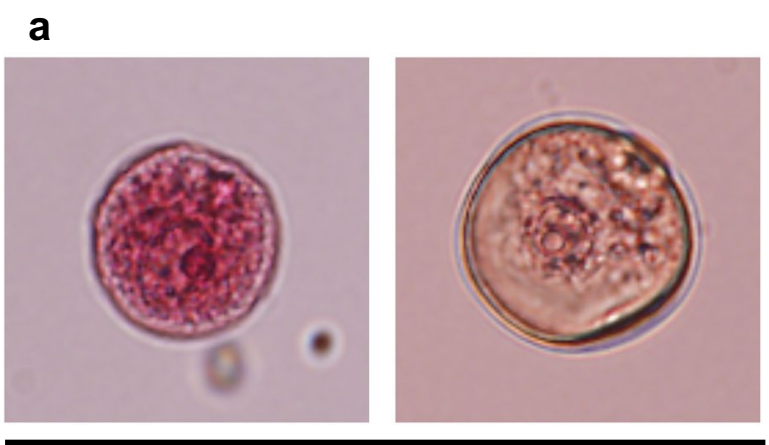

Early $1 \mathrm{~N}$

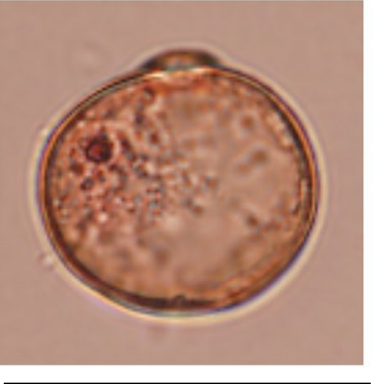

Middle $1 \mathrm{~N}$

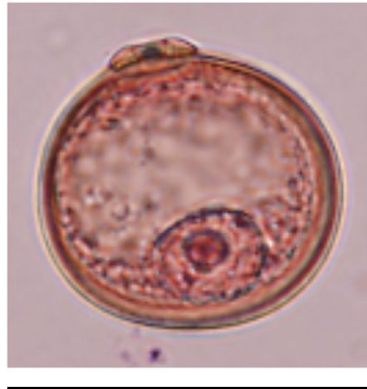

Late $1 \mathrm{~N}$
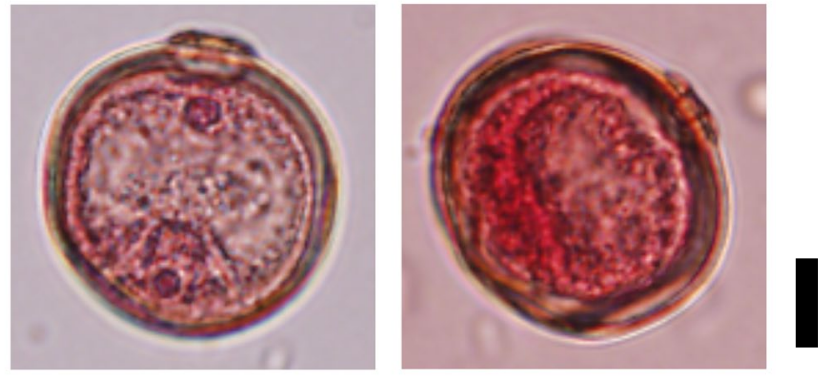

$2 \mathrm{~N} / 3 \mathrm{~N}$

b
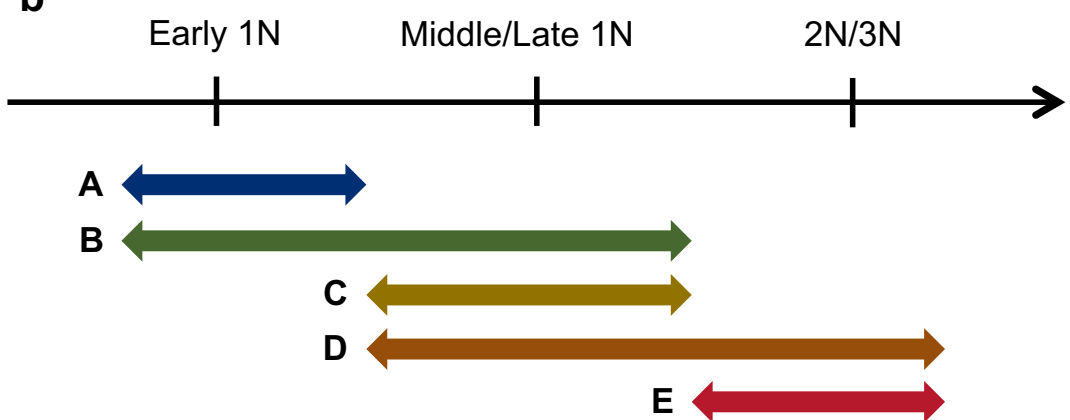

Fig. 1 Classification of microspores based on developmental stages. Developmental stages of microspores were judged by microscopic observation after low temperature treatment, and the classes of anthers or calli were defined on the basis of these stages. a Microspores stained with acetocarmine at different developmental stages. Bar, $10 \mu \mathrm{m}$. b Categorization of microspore developmental stages into classes A to E. 1N, 2N, and $3 \mathrm{~N}$ indicate uninucleate, binucleate, and trinucleate stages, respectively

gamete eliminator locus $S_{1}$ was expected to fit neither of these two ratios.

\section{Linkage mapping of $\mathrm{S}_{1}$ and $\mathrm{S}_{19}$ based on TRD}

Genotype data of calli from GIL/T65 were used for linkage mapping of the partial lethal-factor locus causing TRD [32,33]. The chromosomal positions of $S_{1}$ and $S_{19}$ were calculated according to Cheng et al. [32,33]. Each $H S$ locus was assumed to be adjacent to two SSR markers, one on each side. Recombination values between an $H S$ locus and each of the two SSR markers were designated as $r_{1}$ and $r_{2}$, respectively. The parameter $t$ (where $0<t<1$ ) was used to denote the viability of the killed genotype (sativa-type alleles for $S_{1}$ and $S_{19}$ ) relative to the killer genotype (glaberrima-type alleles for $S_{1}$ and $S_{19}$ ). Recombination values on each interval of any two adjacent markers were used as initial values for $r_{1}$ and $r_{2}$ subject to repeated calculations, and parameter $t$ was initialized to the frequency of non-recombinant sativa-type calli relative to non-recombinant glaberrima-type calli. Calculations were terminated when any of the parameters became zero or the relative errors of all parameters 
became less than $1 \times 10^{-6}$. The $H S$ locus was considered to be located in the interval in which $t$ was minimized and neither $r_{1}$ nor $r_{2}$ were zero. The locations estimated for $S_{1}$ and $S_{19}$ were collated with the mapping results of previous studies [34, 35].

\section{Results}

\section{Callus induction in interspecific $F_{\mathbf{1}}$ hybrids}

We obtained microspore-derived calli from interspecific $\mathrm{F}_{1}$ hybrids whose male gametes were completely sterile because of the lethal effect of multiple $H S$ loci. A total of 104 calli were obtained from 40,092 anthers of WK/ Ns plants plated on CIM (Additional file 2: Table S3). In addition to N6 medium, we used RI-13 and SK-1 media as CIM, both of which have been reported to be suitable for callus formation in O. glaberrima and O. sativa ssp. indica, respectively, but not in O. sativa ssp. japonica cultivars [27-29]. Among the three AC media tested on $\mathrm{F}_{1}$ hybrid lines N/WK21 and WK21/N, RI-13 gave the highest callus formation rates (Additional file 2: Table S3). We therefore used RI-13 as the CIM for the remainder of the study. Of the three parental lines used as controls, the highest callus formation rate on RI-13 CIM was observed in the japonica cultivar Nipponbare $(4.37 \%)$; the two O. glaberrima cultivars, WK21 and WK18, had rates of $1.10 \%$ and $2.12 \%$, respectively. Callus formation rates of WK/Ns were lower than those of the parental lines.

We next determined the optimum microspore developmental stage for callus formation based on classes A to $\mathrm{E}$ (Fig. 1) in $\mathrm{F}_{1}(\mathrm{WK} 21 / \mathrm{N})$ and parental lines. WK21/N and Nipponbare had the highest callus formation rates $(0.93 \%$ and $5.87 \%$, respectively) during the class $C$ period (early $1 \mathrm{~N}$ to middle/late $1 \mathrm{~N}$ stages), and WK21 exhibited high rates during the class $C$ period $(1.99 \%)$ and also from middle/late $1 \mathrm{~N}-2 \mathrm{~N}$ stages (class D) (2.02\%) (Additional file 2: Table S4). As reported for japonica rice cultivars [30], class $C$ (i.e. middle and late $1 \mathrm{~N}$ stages) was typically the most suitable period for efficient callus formation from anthers of $O$. glaberrima and its hybrid with japonica.

\section{Genetic constitutions of HS loci in interspecific hybrid calli} Eleven $H S$ loci are currently known to be HS factors involved in pollen sterility in interspecific $F_{1}$ hybrids between $O$. sativa and O. glaberrima [6-15] (Table 1). Heterozygotes of gamete eliminator loci $S_{1}$ and $S_{37}(t)$ and pollen killer loci $S_{3}, S_{19}$, and $S_{20}$ preferentially transmit O. glaberrima alleles $(g)$ to progenies, whereas heterozygotes of pollen killer locus $S_{21}$ transmit $O$. sativa alleles (s) $[7,16,17,36,37]$.

We genotyped the 104 microspore-derived calli in this study using SSR markers closely linked to known $H S$ loci. Among the 104 calli, 52 were found to be haploid or to be completely homozygous for alleles from either O. sativa or O. glaberrima according to all markers examined, which suggests that these calli were derived from normal microspores after meiosis (Additional file 1: Fig. S2). The remaining 52 calli possessed genomes containing a mixture of homozygous and heterozygous alleles at the examined loci. The incomplete genetic fixation in these calli may have been derived from microspores generated by abnormal meiotic processes, e.g., unreduced gamete formation. These calli were therefore excluded from further analysis of SD. We investigated the segregation of $H S$ alleles of completely homozygous calli by genotyping SSR markers located near each $H S$ locus. Significant SD was observed for more than half of the 11 genotyped $H S$ loci, with the segregation of $S_{1}, S_{3}, S_{19}, S_{20}, S_{21}$, and $S_{29}(t)$ found to be markedly distorted (Table 1 ). Because the segregation pattern of these HS loci was consistent with previous reports described above, the gametophytic effects of some $H S$ loci involved in pollen viability determination were reflected in the genotype frequencies of calli derived from microspores of interspecific $F_{1}$ plants. In contrast, no significant SD was detected for five $H S$ loci, including $S_{37}(t)$, and TRD at these loci should not have occurred at the $1-2 \mathrm{~N}$ microspore developmental stages used for AC.

\section{SD of $\mathrm{S}_{1}$ and $\mathrm{S}_{19}$ caused by pollen semi-sterility}

The SD of $H S$ loci observed in calli from interspecific $\mathrm{F}_{1}$ hybrids resulted from allele-specific abortion of microspores. We therefore considered using genotype data from these calli for rapid mapping of $H S$ loci without the need for multiple generations from crossing experiments. To confirm the feasibility of mapping $H S$ loci using microspore-derived calli, we carried out this strategy with $S_{1}$ and $S_{19}$ loci. Because $S_{1}$ is the major factor influencing HS between $O$. sativa and O. glaberrima and one of the most well-known gamete eliminators, detailed information on the location and function of this locus is available [19, 20,34]. $S_{19}$ is also one of the best-studied pollen killers, and its TRD characterization and fine mapping have been performed [35]. Heterozygotes subjected to AC were prepared from O. glaberrima introgression lines (GILs) in an O. sativa genomic background [26]. Although mainly composed of chromosomes from $O$. sativa T65, the genomes of GIL31 and GIL27 also contained some large pieces of chromosomal fragments from O. glaberrima. GIL31 and GIL27 were homozygous for glaberrima alleles at $S_{1}$ and $S_{19}$ located on chromosomes 6 and 3, respectively (Additional file 1: Fig. S1). To produce $S_{1}$ and $S_{19}$ heterozygotes, GIL31 and GIL27 were respectively crossed with $\mathrm{T} 65$, and the progenies were designated as GIL/T65. 
To confirm the effect of $S_{1}$ and $S_{19}$ in GIL/T65, pollen fertility was examined. GIL31/T65 produced normal pollen grains at a rate of $58.3 \%$, which compares with $92.2 \%$ and $98.0 \%$ for GIL31 and T65, respectively (Fig. 2a). Similarly, the mature pollen grains of GIL27/ T65 were semi-sterile, whereas its parental lines were highly fertile. If two or more $H S$ loci had independently disrupted microspore development, pollen fertility would have been theoretically reduced to less than $25 \%$. Consequently, $H S$ loci other than $S_{1}$ and $S_{19}$ did not appear to be present in GIL31/T65 and GIL27/T65 genomes, respectively. Abortive microspores in GIL31/T65 were readily observed after the late $1 \mathrm{~N}$ stage and were characterized by weak acetocarmine staining, disappearance of contents of the microspores following the progression of developmental stages, and hollow grains at the mature pollen stage (Fig. 2). In contrast, abortive microspores in
GIL27/T65 were not distinguishable from normal ones until the early $2 \mathrm{~N}$ stage and seemed to accumulate less starch in the latter stage of pollen development (Fig. 2a). As far as the microscopic observation was concerned, the gametophytic lethal effect of $S_{1}$ was shown in microspores during the class $\mathrm{C}$, while the effect of $S_{19}$ appeared on the later than the class C. Genotyping of $F_{2}$ plants from GIL31/T65 with the SSR marker RM19359 at the $S_{1}$ locus revealed an apparent distortion of the $S_{1}^{g}$ homozygous allele (54/56 plants) (Additional file 2: Table S5). As revealed by the $F_{2}$ plants, the preferential transmission of the $S_{1}^{g}$ allele to the progenies through both male and female gametophytes validated an earlier finding that the $S_{1}^{g}$ allele is a gamete eliminator causing male and female TRD [6]. In contrast, $S_{1}^{g}$ homozygotes (20/42 plants) were excessively segregated in $\mathrm{F}_{2}$ plants derived from GIL27/ T65, and the 1:1 segregation ratio for gg:sg genotypes
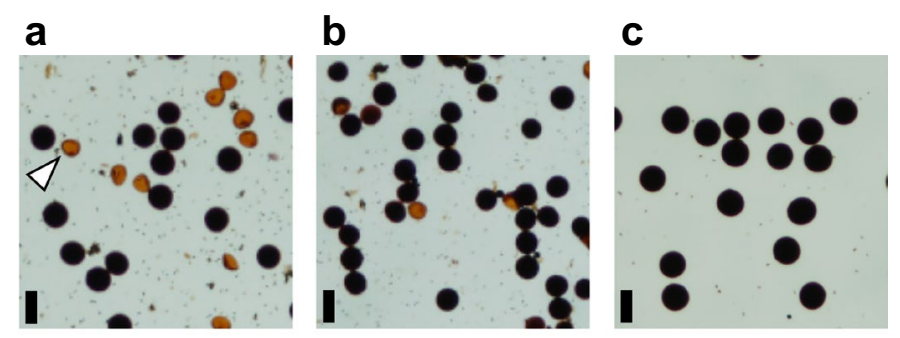

d

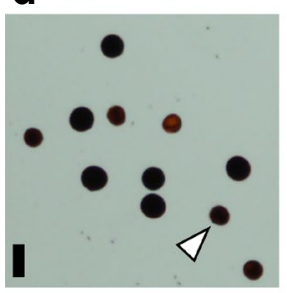

e

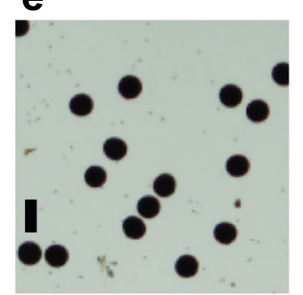

g
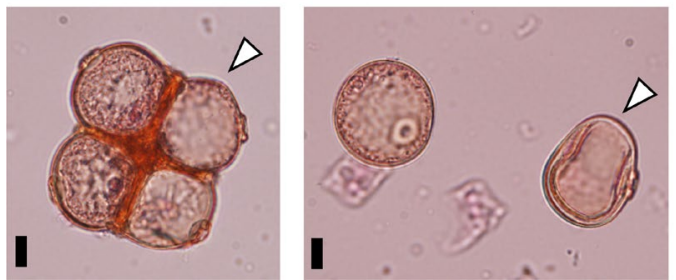
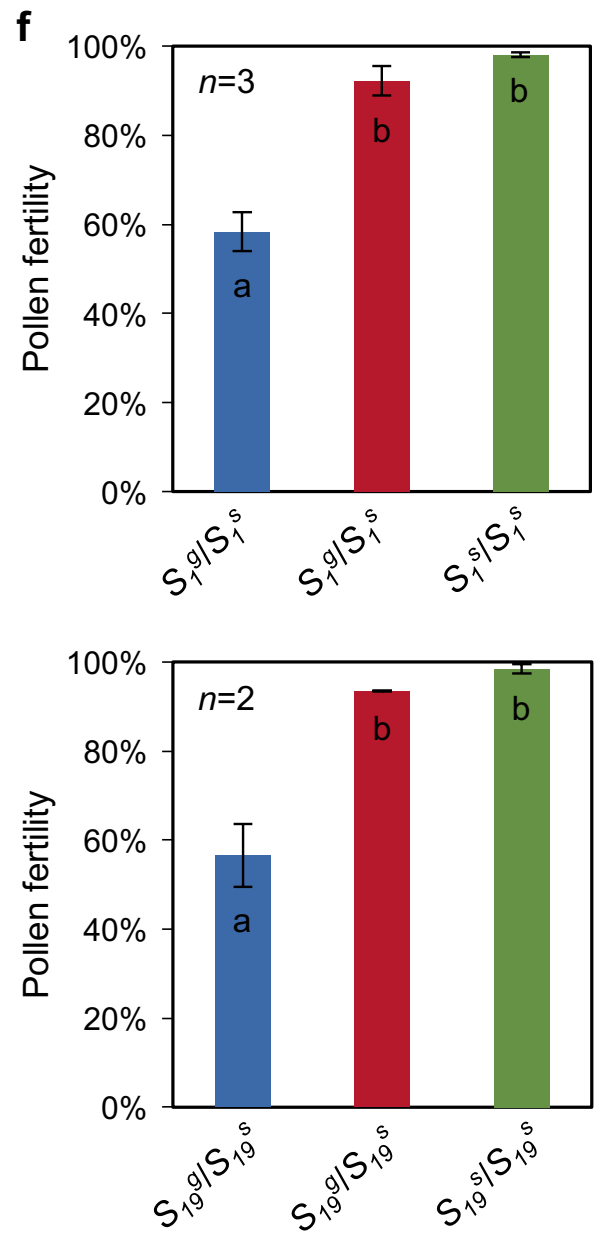

Fig. 2 Microscopic observations of pollen semi-sterility caused by $S_{1}$ and $S_{19}$ in the heterozygous state. a-e Mature pollen stained with $I_{2}-K I$ solution in a $S_{1}^{g} / S_{1}^{s}$ heterozygotes, b $S_{1}^{g}$ homozygotes, c $O$. sativa' Taichung $65^{\prime}\left(S_{1}^{s}\right.$ and $S_{1}^{s}$ homozygotes), d $S_{1}^{g} / S_{1}^{s}$ heterozygotes, and e $S_{19}^{g}$ homozygotes. Bar, $50 \mu \mathrm{m}$. f Pollen fertility exhibited by each genotype of $S_{1}$ and $S_{19}$ loci. Error bars indicate standard errors. Different lowercase letters between bars indicate significant differences at the $5 \%$ level (Welch's $t$ test corrected by Bonferroni's method). $\mathbf{g}$ Aberrant microspores (indicated by the arrowhead) of $S_{1}^{g} / S_{1}^{s}$ heterozygotes stained with acetocarmine at the late uninucleate stage. Bar, $10 \mu \mathrm{m}$ 
at the $S_{19}$ locus suggests that the $S_{19}^{g}$ allele functions as a pollen killer as reported previously (Additional file 2: Table S5) [9].

\section{AC-induced callus formation of GIL/T65 and distortions at $S_{1}$ and $S_{19}$ loci}

In both GIL31/T65 and its parental lines, higher rates of $\mathrm{AC}$-induced callus formation were observed in $\mathrm{C}$ - and D-class microspore stages compared with classes $\mathrm{B}$ and E (Additional file 2: Table S6). No callus was obtained from class A microspore. The maximum rate of callus formation in GIL31/T65 was 16.4\%, which was less than half that of the parental lines. Rates were almost identical between the two parental lines. These results indicate that no locus existed that accounted for the different callus formation efficiencies between $s$ and $g$ alleles within the genomic region including the $S_{1}$ locus. Consequently, the SD observed in this region in the callus population from GIL31/T65 can be considered to be due to TRD by the region including the $S_{1}$ gene. A total of 1685 of the 2299 calli obtained in this study were randomly selected and genotyped with four SSR markers surrounding the $S_{1}$ locus. Most of the genotyped calli possessed the $S_{1}^{g}$ allele, which corresponds to extreme SD towards the $S_{1}^{g}$ type (Table 2). The SD observed in the earlier microspore stage class B was comparable to that in classes C and D. The fates of microspores derived from the $S_{1}^{g} / S_{1}^{s}$ heterozygous parent were thus likely determined before the middle $1 \mathrm{~N}$ stage in an allele-dependent manner.

Calli from GIL27/T65 obtained from class-C microspores accounted for $21.1 \%$ of the total number of cultured anthers (3114; Additional file 2: Table S7). The similar rates of callus formation between the two parents confirms that the SD around the $S_{19}$ locus was due simply to the gametophytic effect of the $S_{19}$ gene. Distortion similar to that seen at the $S_{1}$ locus was observed at the heterozygous $S_{19}$ locus of AC-induced calli of GIL27/ T65 (Table 3). The segregation of RM132 and RM14349, DNA markers adjacent to the $S_{19}$ locus, was significantly distorted by calli carrying the $S_{1}^{g}$ allele. The different SDs of the SSR markers around the two HS loci may have

Table 3 Genotype segregation of markers linked to the $S 19$ locus in anther culture-induced calli derived from $S 19$ heterozygotes

\begin{tabular}{|c|c|c|c|c|}
\hline \multicolumn{5}{|c|}{ No. of callus (frequency) ${ }^{a}$} \\
\hline & \multicolumn{2}{|c|}{ RM132 } & \multicolumn{2}{|c|}{ RM14349 } \\
\hline & $s$ & $g$ & $s$ & $g$ \\
\hline \multicolumn{5}{|l|}{ Class } \\
\hline \multirow[t]{2}{*}{ B } & 4 & 24 & 5 & 24 \\
\hline & $(0.14)$ & $(0.86)$ & $(0.17)$ & $(0.83)$ \\
\hline \multirow[t]{2}{*}{$C$} & 50 & 408 & 56 & 404 \\
\hline & $(0.11)$ & $(0.89)$ & $(0.12)$ & (0.88) \\
\hline \multirow[t]{2}{*}{$D$} & 4 & 20 & 0 & 23 \\
\hline & $(0.17)$ & $(0.83)$ & 0.00 & $(1.00)$ \\
\hline \multirow[t]{2}{*}{ Total } & 58 & 452 & 61 & 451 \\
\hline & $(0.11)$ & $(0.89)$ & $(0.12)$ & (0.88) \\
\hline
\end{tabular}

Class $B$ to $D$ are based on the developmental stage of microspores

a s, O. sativa allele; $\mathrm{g}$, O. glaberrima allele

Table 2 Genotype segregation of markers linked to the S1 locus in anther culture-induced calli derived from S1 heterozygotes

\begin{tabular}{|c|c|c|c|c|c|c|c|c|}
\hline & \multicolumn{8}{|c|}{ No. of callus (frequency) ${ }^{a}$} \\
\hline & \multicolumn{2}{|c|}{ RM7399 } & \multicolumn{2}{|c|}{ RM19359 } & \multicolumn{2}{|c|}{ RM204 } & \multicolumn{2}{|c|}{ RM276 } \\
\hline & $s$ & $g$ & $s$ & $g$ & $s$ & $g$ & $s$ & $g$ \\
\hline \multicolumn{9}{|l|}{ Class } \\
\hline \multirow[t]{2}{*}{ B } & 1 & 24 & 0 & 25 & 1 & 24 & 1 & 24 \\
\hline & $(0.04)$ & $(0.96)$ & 0.00 & $(1.00)$ & $(0.04)$ & $(0.96)$ & $(0.04)$ & $(0.96)$ \\
\hline \multirow[t]{2}{*}{ C } & 67 & 1108 & 4 & 1171 & 52 & 1166 & 270 & 905 \\
\hline & $(0.06)$ & $(0.94)$ & $(0.00)$ & $(1.00)$ & $(0.05)$ & $(0.96)$ & $(0.23)$ & $(0.77)$ \\
\hline \multirow[t]{2}{*}{$\mathrm{D}$} & 6 & 355 & 2 & 361 & 18 & 344 & 80 & 285 \\
\hline & $(0.02)$ & $(0.98)$ & $(0.01)$ & $(0.99)$ & $(0.05)$ & $(0.95)$ & $(0.22)$ & $(0.78)$ \\
\hline \multirow[t]{2}{*}{ E } & 2 & 13 & 2 & 13 & 2 & 13 & 5 & 10 \\
\hline & $(0.13)$ & $(0.87)$ & $(0.13)$ & $(0.87)$ & $(0.13)$ & $(0.87)$ & $(0.33)$ & $(0.67)$ \\
\hline \multirow[t]{2}{*}{ Total } & 76 & 1500 & 8 & 1570 & 73 & 1547 & 356 & 1224 \\
\hline & $(0.05)$ & $(0.95)$ & $(0.01)$ & $(1.00)$ & $(0.05)$ & $(0.96)$ & $(0.23)$ & $(0.78)$ \\
\hline
\end{tabular}

Class $A$ to $D$ are based on the developmental stage of microspores

${ }^{a}$ s: O. sativa allele; g: O. glaberrima allele 
resulted from each genetic recombination frequency. The detection of SD at the class-B microspore stage implies that the TRD associated with $S_{19}$ occurred even earlier than the middle $1 \mathrm{~N}$ microspore stage (Table 3 ).

\section{Fine mapping of $\mathrm{S}_{1}$ and $\mathrm{S}_{19}$ using $\mathrm{AC}$-induced callus genotypes}

The degree of SD, which should be relaxed in proportion to the genetic distance of a marker from a TRDcausative gene, can be plotted on a unimodal chart [18, 32, 33]. The maximum SD at the $S_{1}$ locus was observed at the SSR marker RM19359, with the SD of other markers reduced because they were distant from the causative gene (Fig. 3). This reduction in SD as a function of distance from the $S_{1}$ locus can be explained by meiotic recombination. The differing degrees of SD, which are due to meiotic recombination and reflect the distance between a causative gene and a marker, can be effectively applied for fine mapping of $H S$ loci in a callus population derived from their heterozygotes.

We mapped $S_{1}$ and $S_{19}$ using the genotype data obtained for AC-induced calli from GIL31/T65 and GIL27/T65, respectively, based on the method described by Cheng et al. [33]. The genotype data were analyzed without considering microspore developmental stages because the degree of SD was almost identical among the developmental classes (Tables 2 and 3). The two lethal factors, $S_{1}$ and $S_{19}$, were respectively located at their expected positions on chromosomes 6 and 3 (Fig. 4, Additional file 2: Table S8). The lethal factors for $S_{1}$ on the short arm of chromosome 6 have recently been identified by map-based cloning [19, 20, 34]. Our linkage mapping using the AC-induced calli precisely located

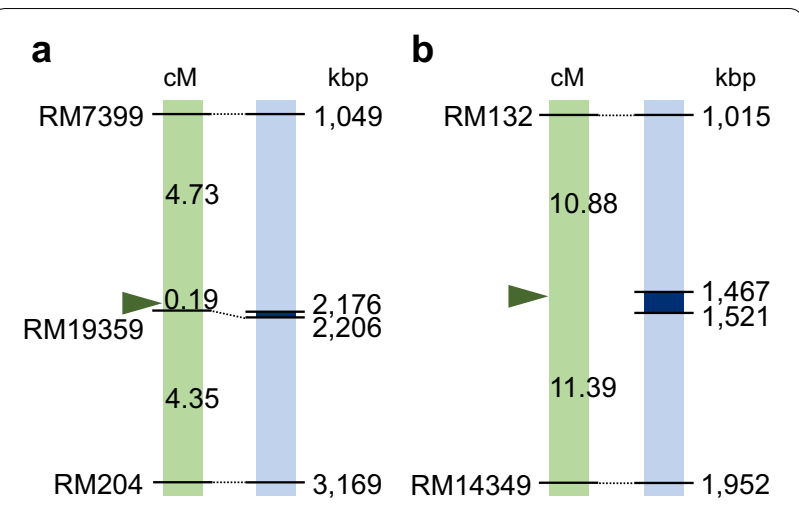

Fig. 4 Linkage analyses of $S_{1}$ and $S_{19}$ based on the genotypes of anther culture-induced calli. The positions of $S_{1}(\mathbf{a})$ and $S_{19}(\mathbf{b})$ loci (green bars), determined according to likelihood estimates (Additional file 2: Table S8) based on TRD from microspore callus induction in this study, are compared with their known positions (blue bars) [34, 35]. Genetic distances were calculated from the recombination parameters using Haldane's mapping function. Arrowheads indicate putative locations of $S_{1}(\mathbf{a})$ and $S_{19}(\mathbf{b})$. Blue bars represent the physical map of the corresponding region based on the Oryza sativa japonica'Nipponbare' reference genome (IRGSP-1.0). The dark blue area on each blue bar represents the region in which $S_{1}(\mathbf{a})$ or $S_{19}(\mathbf{b})$ was mapped $[34,35,42]$

$S_{1}$ between RM7399 and RM19359 on chromosome 6 (Fig. 4a). The probable location of the pollen killer $S_{19}$ was mapped between RM132 and RM14349 on chromosome 3 (Fig. 4b), consistent with a previous study [35]. Both $S_{1}$ and $S_{19}$ loci caused extreme TRD through callus formation because of the low $t$ values at the putative $H S$ loci $\left(0.33 \pm 0.15 \%\right.$ at $S_{1}$ and $1.97 \pm 0.63 \%$ at $\left.S_{19}\right)$. Although SD of the two $S_{19}$ markers, RM132 and RM14349, slightly

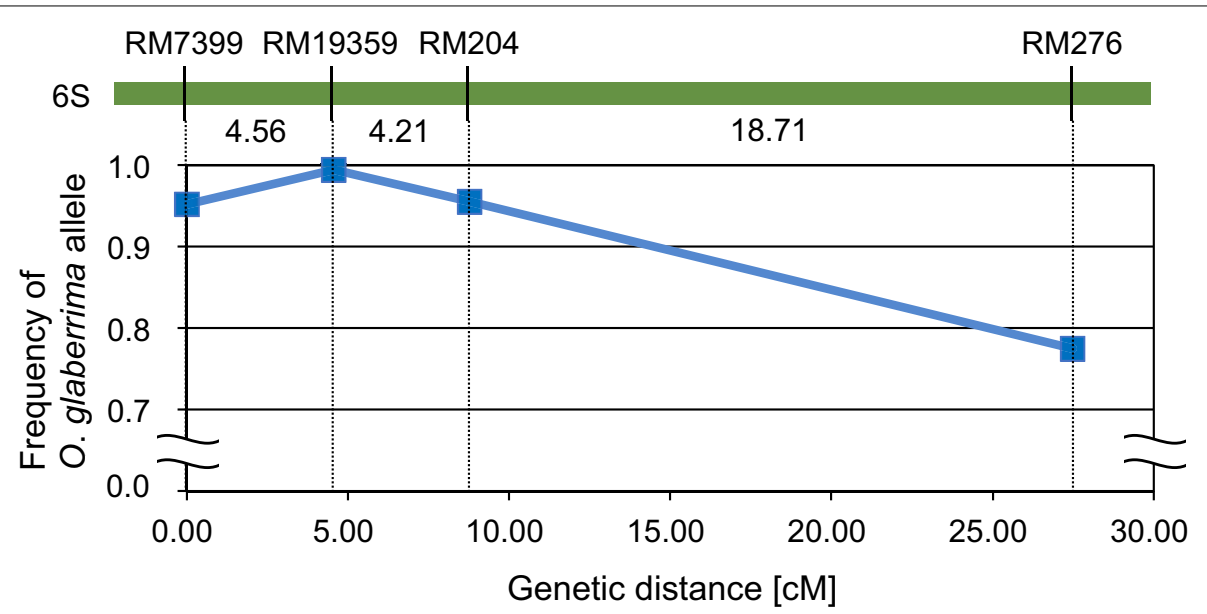

Fig. 3 Unimodal plot of segregation distortion in the $S_{1}$ region in anther culture (AC)-induced calli derived from $S_{1}$ heterozygotes. The frequency of the O. glaberrima allele in AC-induced calli from $S_{\text {, }}$ heterozygotes was detected with simple sequence repeat (SSR) markers (left axis). Genetic distances (below the green line) between SSR markers were calculated from recombination values using Kosambi's mapping function 
recovered depending on their genetic distances from $S_{19}$, the $t$ estimate for $S_{19}$ conformed to the severe SD that has been previously observed [35, 36]. These results demonstrate the applicability of using genotype data from ACinduced calli to construct fine maps for $H S$ loci.

\section{Discussion}

\section{Availability of AC-induced calli for the genetic study of HS}

In this study, we found that $H S$ loci responsible for TRD of male gametophytes can act as segregation distorters in AC-induced calli (Table 1). This gametophytic effect was observed to directly influence the frequency of genotypes in AC-induced calli arising from $H S$-locus heterozygotes. This characteristic makes AC-induced callus a useful material to analyze TRD of male gametes involved in HS. Genetic analysis using an AC-induced callus population can accelerate the detection of $H S$ loci and greatly reduce the amount of time required for mapping $H S$ loci compared with the conventional backcrossing method (Fig. 5). When the conventional method is used, the $F_{1}$ sativa-glaberrima offspring must be backcrossed as a female parent to either of the parental strains because of complete male sterility due to interactions at a dozen of $H S$ loci. The male sterility-related phenotype caused by the $H S$ locus appears at the end of reproductive organ development in $\mathrm{BC}_{1} \mathrm{~F}_{1}$ plants, and TRD of male gametes can be detected no earlier than the next generation (Fig. 5).

In this study, we simultaneously detected $\mathrm{SD}$ of six $H S$ loci, namely, $S_{1}, S_{3}, S_{19}, S_{20}, S_{21}$, and $S_{29}(t)$, in anthercultured calli induced from microspores of WK/Ns, the hybrids between $O$. sativa and $O$. glaberrima. These instances of SD detected in the single AC callus population can be regarded as examples of TRD occurring during microspore development. Nevertheless, SD in a callus population might not always be linked to $H S$ loci, but may sometimes be influenced by other factors, such as callus-formation efficiency. In previous studies using japonica-indica hybrids, distorted chromosomal regions were compared between $\mathrm{F}_{2}$ plants and $\mathrm{AC}$ doubledhaploid plants derived from the same $F_{1}$ plants [23-25]. These comparisons revealed that the doubled-haploid plant population gave rise to different kinds of genomic distortions; some loci underwent SD in both the doubledhaploid plant population and $\mathrm{F}_{2}$ plants, whereas the $\mathrm{SD}$ of other loci was unique to doubled-haploid plants. These different cases of SD may have been due to various causes of TRD, such as gametophytic interactions in the hybrids, different efficiencies in the process of callus induction from microspores, or plant regeneration from callus. To confirm whether the TRD of HS loci was detectable in the interspecific $\mathrm{F}_{1} \mathrm{AC}$ calli, further experiments were therefore performed using two CSSLs containing either
$S_{1}$ or $S_{19}$ segments. The degree of SD uncovered by these experiments precisely reflected the distance from the position of each $H S$ locus (Tables 2, 3; Fig. 3). The location of a potential $H S$ locus can be inferred from loci exhibiting excessive TRD based on the segregation of a single marker or likelihood estimation using linked markers. In this study, the use of AC-induced calli from interspecific hybrids allowed us to estimate the location of an $H S$ locus by analyzing the degree of TRD using DNA markers comprising a linkage group surrounding the locus.

HS is assumed to be due to the accumulated effects of multiple $H S$ loci $[5,18]$. In intraspecific hybrids, an $H S$ locus that causes sterility is dependent on the cross combination and can be discovered from various crossing experiments [38, 39]. The conventional method used for detection and mapping of $H S$ loci requires at least three generations to establish initial $\mathrm{BC}_{1} \mathrm{~F}_{2}$ materials, with more laborious work needed thereafter to sort out each $H S$ locus (Fig. 5). In contrast, the AC method allowed us to comprehensively screen and map loci causing TRD of male gametes in the interspecific $F_{1}$ population just after the first hybridization. The genotype segregation of AC-induced calli can be effectively used for rapid detection of HS loci causing TRD. Moreover, this strategy can be applied to other crops if in vitro callus formation or embryogenesis from microspores is possible. However, the efficiency of callus induction is considered to be a limiting factor to evaluate TRD. The interspecific hybrids may cause low frequency of callus induction unable to appropriately map TRD factors.

\section{Relationship between SD and microspore development}

AC-induced calli should lose their identity as microspores and possess characteristics similar to vegetative tissue (e.g. roots) [40, 41]. The deleterious effect of $H S$ genes should appear only in reproductive tissue involved in gametogenesis. Consequently, $H S$ genes are unlikely to function in microspores after the initiation of callus induction. Pollen semi-sterility caused by gametophytic lethal effects of $S_{1}$ and $S_{19}$ have been previously reported to occur at the $2 \mathrm{~N}$ stage or even later $[17,35]$. In the present study, our microscopic observations suggested that microspore abortion caused by $S_{1}$ and $S_{19}$ occurred after the late $1 \mathrm{~N}$ and $2 \mathrm{~N}$ stages, respectively, whereas the pattern of SD due to $S_{1}$ and $S_{19}$ in AC-induced calli from GIL/T65 provided evidence for the occurrence of allele-specific abortion prior to the $1 \mathrm{~N}$ stage (Tables 2 and 3). Our results using the AC method suggest that gametophytic lethal effects of $S_{1}$ and $S_{19}$ genes arose during early microspore development. In contrast to six $H S$ loci, namely, $S_{1}, S_{3}, S_{19}, S_{20}, S_{21}$, and $S_{29}(t)$, that displayed SD in AC-induced calli, five $H S$ loci, namely, $S_{18}$, 


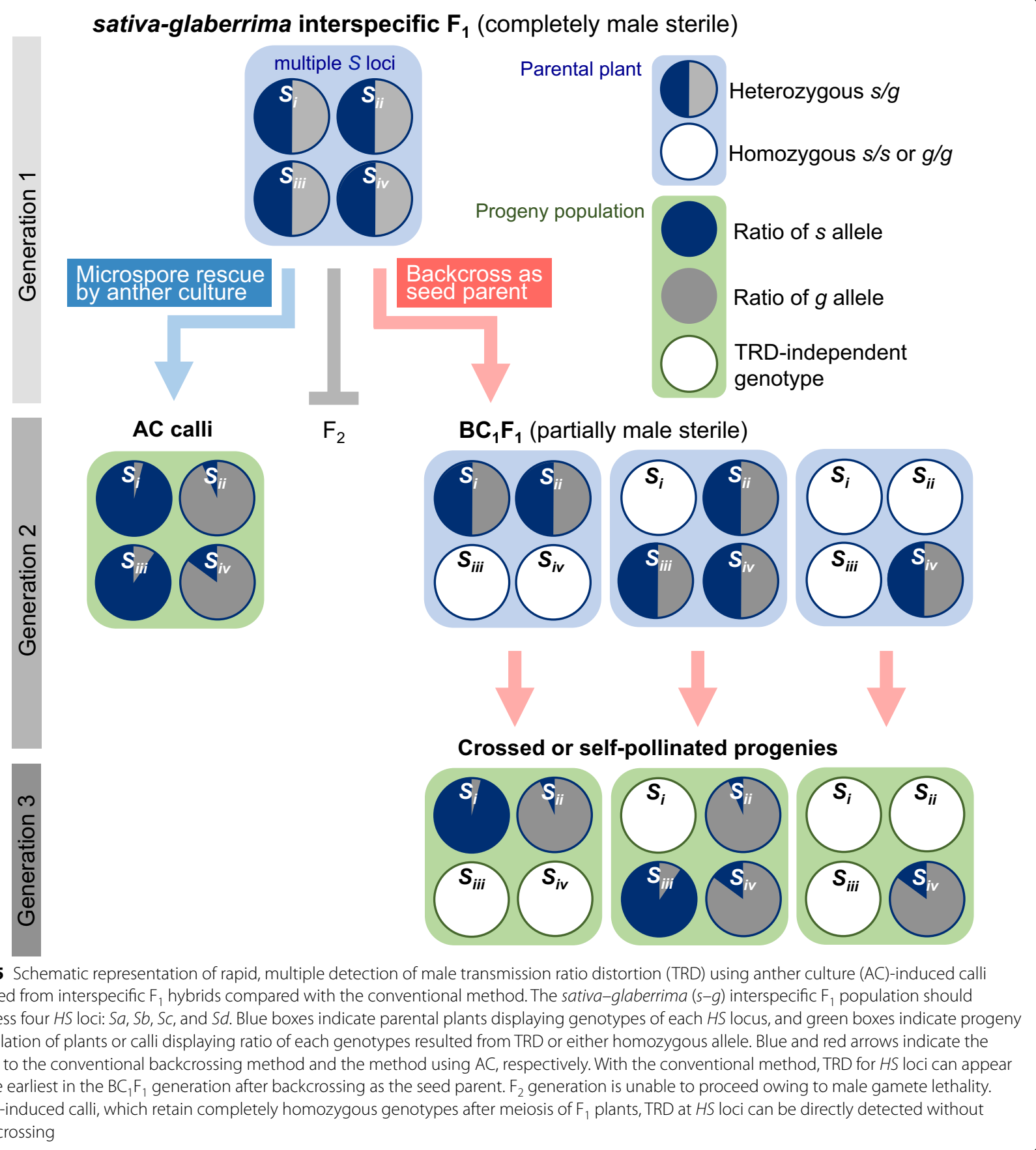

$S_{34}(t), S_{36}(t), S_{37}(t)$, and $S_{38}(t)$, underwent no significant $\mathrm{SD}$ in AC-induced calli from WK/Ns (Table 1). Several different reasons can be invoked to explain why these five $H S$ loci did not exhibit SD in AC-induced calli: (1) allele-specific abortion may have taken place after the $2 \mathrm{~N}$ stage of microspore development; (2) no SD occurred, but pollen sterility was caused by a sporophytic effect; or (3) the HS-locus antagonistic relationships detected in sativa-glaberrima hybrids did not occur between Nipponbare and WK21.

\section{Conclusions}

Taken together, our results demonstrate that the genotype segregation patterns of $H S$ loci in AC-induced calli associated with microspore developmental stages are informative for understanding when $H S$ loci determine 
the viability of male gametes. In this study, we have provided the first evidence that $S_{1}$ and $S_{19}$ genes initiate gametophytic pollen abortion before the middle $1 \mathrm{~N}$ stage. Observations based on callus induction of microspores should not only accelerate genetic analysis of $H S$ loci but also deepen our understanding of their functional characteristics.

\section{Additional files}

Additional file 1: Figure S1. Chromosomal positions of O. glaberrima fragments in GIL31 (a) and GIL27 (b). Boxes represent chromosomal segments of GIL31 and GIL27 based on genotype data obtained from Oryzabase (https://shigen.nig.ac.jp/rice/oryzabase/). The different colored regions indicate homozygous Taichung65 fragments (white), homozygous IRGC104038 fragments (blue), and heterozygous or undetermined fragments (gray). The black bars on the right sides of boxes indicate homozygous IRGC104038 fragments on chromosome 6 of GIL31 (a) and chromosome 3 of GIL27 (b) harboring $S_{1}$ and $S_{19}$, respectively. Figure S2. Graphical representation of genotypes of calli derived from interspecific $F_{1}$ hybrids between $O$. sativa and O. glaberrima. A total of 104 anther culture-induced calli were evaluated using 11 simple sequence repeat (SSR) markers linked to HS loci; 52 calli were completely homozygous at all markers, and 52 exhibited partial heterozygosity. Cell colors indicate allele genotypes at the 11 SSR loci as follows: yellow, homozygous for the glaberrima allele; green, homozygous for the sativa allele; red, heterozygous.

Additional file 2: Tabe S1. Composition of callus induction media. (a) N6; (b) RI-13; (c) SK-1. Tabe S2. SSR markers for genotyping of HS loci. Tabe S3. Summary of the results of callus induction by anther culture of interspecific $F_{1}$ hybrids between $O$. sativa and $O$. glaberrima. Tabe $\mathbf{S} 4$. Callus formation rates from anther culture of interspecific $F_{1}$ hybrids between $O$. sativa and $O$. glaberrima. Tabe $\mathbf{S 5}$. Segregation distortion in self-pollinated progenies of $S_{1}$ and $S_{19}$ heterozygotes. Tabe $\mathbf{S 6}$. Callus formation rates from anther culture of $S$, heterozygotes. Tabe $\mathbf{S}$. Callus formation rates from anther culture of $S_{19}$ heterozygotes. Tabe $\mathbf{S 8}$. Maximum likelihood estimates of recombination rates and viability parameters.

\section{Abbreviations}

AC: anther culture (AC); HS: hybrid sterility (HS); CSSLs: chromosome segment substitution lines (CSSLs); TRD: transmission ratio distortion (TRD); SD: segregation distortion (SD); N/WK21: $F_{1}$ line between Nipponbare (seed parent) and WK21 (pollen parent); WK21/N: $\mathrm{F}_{1}$ line between WK21 (seed parent) and Nipponbare (pollen parent); N/W18: $F_{1}$ line between Nipponbare (seed parent) and WK18 (pollen parent); WK18/N: $F_{1}$ line between WK18 (seed parent) and Nipponbare (pollen parent); WK/N: collective name for N/WK21, WK21/N, N/ WK18, WK18/N; T65: Taichung 65; GILs: O. glaberrima introgression lines; GIL31/ T65: GIL31 backcrossed with T65 (and, respectively) and; GIL27/T65: GIL27 backcrossed with T65; GIL/T65: collective name for GIL31/T65 and GIL27/T65; CIM: callus-induction medium; SSR: simple sequence repeat.

\section{Authors' contributions}

YKa and YKi planed and designed the research. YKa, DK, and El performed experiments. YKa and YKo analyzed data. YO and HY arranged materials. YKa and YKi wrote the manuscript. All authors read and approved the final manuscript.

\footnotetext{
Author details

${ }^{1}$ Laboratory of Plant Breeding, Research Faculty of Agriculture, Hokkaido University, Sapporo 060-8589, Japan. ${ }^{2}$ Laboratory of Plant Breeding, Rakuno Gakuen University, Bunkyodai-Midorimachi, Ebetsu 069-8501, Japan. ${ }^{3}$ Plant Breeding Laboratory, Faculty of Agriculture, Kyushu University, 744 Motooka Nishi-ku, Fukuoka, Japan.
}

\section{Acknowledgements}

The O. glaberrima introgression lines, GIL27 and GIL31, were distributed from Kyushu University supported by the National Bioresource Project (NBRP), AMED, Japan. The authors wish to thank Drs. Atsushi Yoshimura and Yoshiyuki Yamagata (Plant Breeding Laboratory, Kyushu University) and Dr Itsuro Takamure (Laboratory of Plant Breeding, Hokkaido University) for valuable comments on the materials and manuscript. We thank Edanz Group (www.edanz editing.com/ac) for editing the English text of a draft of this manuscript.

\section{Competing interests}

The authors declare that they have no competing interests.

\section{Availability of data and materials}

The datasets used and/or analyzed during the current study are available from the corresponding author on reasonable request.

\section{Consent for publication}

Not applicable.

\section{Publisher's Note}

Springer Nature remains neutral with regard to jurisdictional claims in published maps and institutional affiliations.

Received: 30 August 2018 Accepted: 12 November 2018

Published online: 17 November 2018

\section{References}

1. Niizeki H, Oono K. Induction of haploid rice plant from anther culture. Proc Jpn Acad. 1968;44:554.

2. Guha S, Maheshwari SC. In vitro production of embryos from anthers of datura. Nature. 1964;204:497.

3. Widmer A, Lexer C, Cozzolino S. Evolution of reproductive isolation in plants. Heredity. 2009;102:31-8.

4. Morinaga T, Kuriyama H. Cytogenetical studies on Oryza sativa L. IV. The F1 hybrid of O. sativa L. and O. glaberrima Steud. Jpa J Breed. 1957;7:57-65.

5. Oka HI. Genic analysis for the sterility of hybrids between distantly related varieties of cultivated rice. J Genet. 1957;55:397-409.

6. Sano Y, Chu YE, Oka HI. Genetic-studies of speciation in cultivated rice. 1. Genic analysis for the $\mathrm{F} 1$ sterility between $\mathrm{O}$ sativa $\mathrm{L}$ and Oryza glaberrima Steud. Jpn J Genet. 1979;54:121-32.

7. Sano Y. Genetic studies of speciation in cultivated Rice. 3. A new gene controlling sterility in F1 hybrids of 2 cultivated rice species - its association with photoperiod sensitivity. J Hered. 1983;74:435-9.

8. Doi K, Taguchi K, Yoshimura A. A new locus affecting high F1 pollen sterility found in backcross progenies of Japonica rice and African rice. Rice Genet Newsl. 1998;15:146-147.

9. Taguchi K, Doi K, Yoshimura A. RFLP mapping of S19, a gene for F1 pollen semi-sterility found in backcross progeny of Oryza sativa and O. glaberrima. Rice Genet Newsl. 1999;16:70.

10. Doi K, Taguchi H, Yoshimura A. RFLP mapping of $S 20$ and $S 21$ for F1 pollen semi-sterility found in backcross progeny of Oryza sativa and O. glaberrima. Rice Genet Newsl. 1999;16:65-8.

11. Hu FY, Xu P, Deng XN, Zhou JW, Li J, Tao DY. Molecular mapping of a pollen killer gene S29(t) in Oryza glaberrima and co-linear analysis with S22 in O. glumaepatula. Euphytica. 2006;151:273-8.

12. Ren G, Xu P, Deng X, Zhou J, Hu F, Li J, Li F, Zhang Z, Tao D. A new gamete eliminator from Oryza glaberrima. Rice Genet Newsl. 2006;22:43-4.

13. Zhang Z, Xu P, Hu F, Zhou J, Li J, Deng X, Ren G, Li F, Tao D. A new sterile gene from Oryza glaberrima on chromosome 3. Rice Genet Newsl. 2006;22:26-8.

14. Li F, Liu FH, Morinaga D, Zhao Z. A new gene for hybrid sterility from a cross between Oryza sativa and O. glaberrima. Plant Breed. 2011:130:165-71.

15. Xu P, Zhou JW, Li J, Hu FY, Deng XN, Feng SF, Ren GY, Zhang Z, Deng W, Tao DY. Mapping three new interspecific hybrid sterile loci between Oryza sativa O. glaberrima. Breed Sci. 2014;63:476-82. 
16. Yu X, Zhao Z, Zheng X, Zhou J, Kong W, Wang P, Bai W, Zheng H, Zhang H, $L i J$, et al. A selfish genetic element confers non-Mendelian inheritance in rice. Science. 2018;360:1130-2.

17. Koide Y, Onishi K, Nishimoto D, Baruah AR, Kanazawa A, Sano Y. Sexindependent transmission ratio distortion system responsible for reproductive barriers between Asian and African rice species. New Phytol. 2008; 179:888-900.

18. Garavito A, Guyot R, Lozano J, Gavory F, Samain S, Panaud O, Tohme J, Ghesquiere A, Lorieux M. A genetic model for the female sterility barrier between Asian and African cultivated rice species. Genetics. 2010;185:U1425-39.

19. Koide Y, Ogino A, Yoshikawa T, Kitashima Y, Saito N, Kanaoka Y, Onishi K, Yoshitake Y, Tsukiyama T, Saito H, et al. Lineage-specific gene acquisition or loss is involved in interspecific hybrid sterility in rice. Proc Natl Acad Sci USA. 2018;115:E1955-62.

20. Xie Y, Xu P, Huang J, Ma S, Xie X, Tao D, Chen L, Liu YG. Interspecific hybrid sterility in rice Is mediated by OgTPR1 at the $S 1$ locus encoding a peptidase-like protein. Mol Plant. 2017;10:1137-40.

21. Harushima Y, Nakagahra M, Yano M, Sasaki T, Kurata N. A genome-wide survey of reproductive barriers in an intraspecific hybrid. Genetics. 2001;159:883-92.

22. Harushima Y, Nakagahra M, Yano M, Sasaki T, Kurata N. Diverse variation of reproductive barriers in three intraspecific rice crosses. Genetics. 2002:160:313-22.

23. Guiderdoni E. Gametic selection in anther culture of rice (Oryza sativa L). Theor Appl Genet. 1991;81:406-12.

24. Yamagishi M, Yano M, Fukuta Y, Fukui K, Otani M, Shimada T. Distorted segregation of RFLP markers in regenerated plants derived from anther culture of an F-1 hybrid of rice. Genes Genet Syst. 1996;71:37-41.

25. XuY, Zhu L, Xiao J, Huang N, McCouch SR. Chromosomal regions associated with segregation distortion of molecular markers in F2, backcross, doubled haploid, and recombinant inbred populations in rice (Oryza sativa L). Mol Gen Genet. 1997;253:535-45.

26. Doi K, Iwata N, Yoshimura A. The construction of chromosome substitution lines of African rice (Oryza glaberrima Steud.) in the background of Japonica rice (O. sativa L.). Rice Genet Newsl. 1997;14:39-41.

27. Chu CC. The N6 medium and its applications to anther culture of cereal crops. In: Proceedings of symposium on plant tissue culture. Peking. Science Press; 1978. P. 43-50.

28. Woo SC, Mok T, Huang JY. Anther culture of Oryza sativa L and Oryza perennis Moench Hybrids. Bot Bull Acad Sin. 1978;19:171-8.

29. Raina SK, Zapata FJ. Enhanced anther culture efficiency of indica rice (Oryza sativa L.) through modification of the culture media. Plant Breed. 1997;116:305-15.
30. Kinoshita A, Okamoto Y, Ishimura S, Satake T. Determination of optimum pollen development stage for inducing callus in anther culture of rice. Breed Res. 2000;2:73-9.

31. Martin C, Carpenter R, Sommer H, Saedler H, Coen ES. Molecular analysis of instability in flower pigmentation of Antirrhinum majus, following Isolation of the Pallida locus by transposon tagging. EMBO J. 1985;4:1625-30.

32. Cheng R, Saito A, Takano Y, Ukai Y. Estimation of the position and effect of a lethal factor locus on a molecular marker linkage map. Theor Appl Genet. 1996:93:494-502.

33. Cheng R, Kleinhofs A, Ukai Y. Method for mapping a partial lethal-factor locus on a molecular-marker linkage map of a backcross and doubledhaploid population. Theor Appl Genet. 1998;97:293-8.

34. Guyot R, Garavito A, Gavory F, Samain S, Tohme J, Ghesquiere A, Lorieux M. Patterns of sequence divergence and evolution of the $S-1$ orthologous regions between Asian and African cultivated rice species. PLOS ONE. 2011;6:17726

35. Zhang $Y H$, Zhao ZG, Zhou JW, Jiang L, Bian XF, Wang Y, Wang $C L$, Zhong ZZ, Wang JK, Tao DY, et al. Fine mapping of a gene responsible for pollen semi-sterility in hybrids between Oryza sativa L. and O. glaberrima Steud. Mol Breeding. 2011;28:323-34.

36. Doi K, Taguchi K, Yoshimura A. Genetic analysis of pollen sterility loci found in hybrid progeny between Oryza sativa and O. glaberrima. In: Advances in Rice Genetics. Edited by IRRI. Manila: IRRI; 2003. p. 149-51.

37. Shen YM, Zhao ZG, Ma HY, Bian XF, Yu Y, Yu XW, Chen HY, Liu LL, Zhang $W W$, Jiang $L$, et al. Fine mapping of $S 37$, a locus responsible for pollen and embryo sac sterility in hybrids between Oryza sativa L. and O. glaberrima Steud. Plant Cell Rep. 2015;34:1885-97.

38. Ikehashi $\mathrm{H}$, Araki H. Genetics of F1 sterility in remote crosses of rice. In: Rice genetics. Edited by IRRI. Manila: IRRI; 1986, p. 119-30.

39. Chen JJ, Ding JH, Ouyang YD, Du HY, Yang JY, Cheng K, Zhao J, Qiu SQ, Zhang $\mathrm{XL}$, Yao $\mathrm{JL}$, et al. A triallelic system of $\mathrm{S} 5$ is a major regulator of the reproductive barrier and compatibility of indica-japonica hybrids in rice. Proc Natl Acad Sci USA. 2008;105:11436-41.

40. Ikeuchi M, Sugimoto K, Iwase A. Plant callus: mechanisms of induction and repression. Plant Cell. 2013:25:3159-73.

41. Li H, Soriano M, Cordewener J, Muino JM, Riksen T, Fukuoka H, Angenent $\mathrm{GC}$, Boutilier $\mathrm{K}$. The histone deacetylase inhibitor trichostatin A promotes totipotency in the male gametophyte. Plant Cell. 2014;26:195-209.

42. Yamagishi M, Otani M, Higashi M, Fukuta Y, Fukui K, Yano M, Shimada T. Chromosomal regions controlling anther culturability in rice (Oryza sativa L.). Euphytica. 1998;103:227-34.

\footnotetext{
Ready to submit your research? Choose BMC and benefit from:

- fast, convenient online submission

- thorough peer review by experienced researchers in your field

- rapid publication on acceptance

- support for research data, including large and complex data types

- gold Open Access which fosters wider collaboration and increased citations

- maximum visibility for your research: over $100 \mathrm{M}$ website views per year
}

At BMC, research is always in progress.

Learn more biomedcentral.com/submissions 